\title{
A History of Cerebrovascular Disease Is Independently Associated with Increased Morbidity and Mortality in Patients with COVID-19: A Cohort Study of 369,563 COVID-19 Cases in the USA
}

\author{
Anna M. Nia ${ }^{a}$ Visish M. Srinivasan ${ }^{b}$ Miranda K. Hayworth ${ }^{a}$ Rishi R. Lalla \\ Peter Kan ${ }^{a}$ \\ aDepartment of Neurosurgery, University of Texas Medical Branch, Galveston, TX, USA; ${ }^{\text {b Department of }}$ \\ Neurosurgery, Barrow Neurological Institute, Phoenix, AZ, USA
}

\section{Keywords}

COVID-19 $\cdot$ Cerebrovascular disease $\cdot$ Subarachnoid hemorrhage · Intracerebral hemorrhage · Cerebrovascular complication

\begin{abstract}
Objectives: We set out to evaluate the risk for severe coronavirus disease 2019 (COVID-19) infection and subsequent cerebrovascular disease (CVD) in the population with a prior diagnosis of CVD within the past 10 years. Methods: We utilized the TriNetX Analytics Network to query 369,563 COVID-19 cases up to December 30, 2020. We created 8 cohorts of patients with COVID-19 diagnosis based on a previous diagnosis of CVD. We measured the odds ratios, relative risks, risk differences for hospitalizations, ICU/critical care services, intubation, mortality, and CVD recurrence within 90 days of COVID-19 diagnosis, compared to a propensity-matched cohort with no prior history of CVD within 90 days of COVID-19 diagnosis. Results: 369,563 patients had a confirmed diagnosis of COVID-19 with a subset of 22,497 (6.09\%) patients with a prior diagnosis of CVD within 10 years. All cohorts with a CVD diagnosis had an increased risk of hospitalization, crit-
\end{abstract}

karger@karger.com

(c) 2021 S. Karger AG, Basel

www.karger.com/ced

Karger $\stackrel{2}{5}$ ical care services, and mortality within 90 days of COVID-19 diagnosis. Additionally, the data demonstrate that any history of CVD is associated with significantly increased odds of subsequent CVD post-COVID-19 compared to a matched control. Conclusions: CVD, a known complication of COVID-19, is more frequent in patients with a prior history of CVD. Patients with any previous diagnosis of CVD are at higher risks of morbidity and mortality from COVID-19 infection. In patients admitted to the ED due to COVID-19 symptoms, these risk factors should be promptly identified as delayed or missed risk stratification and could lead to an ineffective and untimely diagnosis of subsequent CVD, which would lead to protracted hospitalization and poor prognosis.

(c) 2021 S. Karger AG, Basel

\section{Introduction}

Coronavirus disease 2019 (COVID-19) caused by the SARS-CoV-2 virus has caused 2.9 million deaths globally as of April 13, 2021, with 135 million cumulative cases reported worldwide [1]. The virus has been detected in CNS tissue samples of a COVID-19 patient who present- 
ed with encephalopathy and altered mental status [2]. The extent of CNS involvement can be influenced by various factors such as genetic predisposition, comorbidities, and environmental factors, which can increase COVID-19 patient's risk of adverse outcomes. Coronaviruses have been shown to invade vascular endothelial cells, which allows the viral particles to directly access CNS neural and glial cells, resulting in a myriad of pathological cerebrovascular changes [3-7]. A case series study by Mao et al. [8] showed that patients who developed severe COVID-19 infection according to their respiratory status had neurologic manifestations, such as acute cerebrovascular diseases (CVDs). Additionally, a retrospective multicenter study by Kummer et al. [9] demonstrated that a history of stroke was associated with inhospital mortality in COVID-19 patients. This can partly be due to intensified inflammatory response and/or immunosuppression involved in all stages of stroke, including poststroke [10, 11]. To our knowledge, there are limited data on whether there is a causal relationship between COVID-19 and subsequent CVD, as well as whether patients with a prior diagnosis of CVD are more likely to experience severe COVID-19 disease manifestations with increased morbidity and mortality. In this cohort study, we assessed whether patients with a history of CVD are at higher risk of severe COVID-19 infection, morbidity, mortality, and CVD recurrence.

\section{Materials and Methods}

\section{Data and Study Design}

The TriNetX Analytics Network, a global federated network that is comprised of de-identified electronic health records from 55 health-care organizations (HCOs), was used for these analyses. The data within TriNetX are provided by HCOs, including academic medical centers and community hospitals in the USA. Available data include demographics, diagnoses, procedures, medications, and laboratory measurement. To comply with legal frameworks and ethical guidelines guarding against data reidentification, the identity of HCOs and their individual contribution to each dataset is not disclosed. The TriNetX platform only uses aggregated counts and statistical summaries of de-identified information. Thus, no protected health information or personal data are made available to the users of the platform. The data are updated daily, and the analyses are performed on the data queries submitted via the browser.

Our inclusion criteria consisted of a confirmed diagnosis of COVID-19 $(369,563)$ in patients older than 18 years up to December 30, 2020. COVID-19 status was determined using codes in EMRs or a positive test result identified with COVID-19-specific laboratory codes. Patients with COVID-19 were identified based on the Centers for Disease Control and Prevention (CDC) coding guidelines ICD-10-CM U07.1 COVID-19 or a positive
COVID-19 RNA nucleic acid amplification test result [12]. The patient population was divided into cohorts based on a prior diagnosis of CVD within the past 10 years. To avoid overgeneralization and elucidate the differences, different diagnosis of CVDs was analyzed as individual cohorts. In particular, cohorts consisted of (1) nontraumatic subarachnoid hemorrhage (I60); (2) nontraumatic intracerebral hemorrhage (I61); (3) other and unspecified nontraumatic intracranial hemorrhage (I62); (4) cerebral infarction (I63), (5) occlusion and stenosis of precerebral arteries (i.e., extracranial carotid and vertebral arteries), not resulting in cerebral infarction (I65); (6) occlusion and stenosis of cerebral arteries, not resulting in cerebral infarction (I66); (7) other CVD (I67); and (8) a control cohort with no prior diagnosis of CVD.

\section{Statistical Analysis}

We identified a set of established comorbidities and risk factors for COVID-19, as follows: age, sex, race (White, Black/African American, and Asian), ethnicity (Hispanic/Latino, Not Hispanic/ Latino, and unknown), essential/primary hypertension, type 2 diabetes mellitus, nicotine dependence, overweight/obesity, chronic kidney disease, atrial fibrillation/flutter, and asthma [13-16]. To assess the prior CVD as a risk factor for severe COVID-19 infection, matched cohorts of patients with the same baseline characteristics, risk factors, and comorbidities were created. The control cohort (w/o history of CVD in the past 10 years) was 1:1 propensity score-matched to each of the 7 cohorts with a previous diagnosis of CVD using the risk factors mentioned above for outcome analyses [17]. Group comparisons for baseline characteristics, hospitalizations, ICU care services, intubation (endotracheal, emergency procedure), mortality, and subsequent CVD within 90 days of COVID-19 diagnosis were made using odds ratios (ORs), relative ratio, and risk difference using the TriNetX platform. The ztest was used to evaluate the statistical significance of the risk difference. For groups with zero patients with adverse outcomes in the matched control cohort with a significant risk difference in the $p$ value, ORs are reported as inf. Statistical significance was set at 2 -sided $p \leq 0.05$. The outcomes are aggregated as Boolean values and allow to assess whether the outcome occurred; however, it does not indicate the number of instances of an outcome during the 90-day period. The study followed the STROBE guidelines [18].

\section{Results}

\section{Analysis of Baseline Characteristics}

A total of 369,563 patients with a confirmed diagnosis of COVID-19 were included in the study. Of these, 22,497 (6.09\%) patients had a prior CVD diagnosis within the past 10 years. As mentioned previously, the patients were separated into 8 cohorts with COVID-19, including 7 cohorts with a diagnosis of COVID-19, a CVD within 10 years, and a matched control COVID-19 cohort without a history of CVD. Table 1 delineates the baseline characteristics for the individual CVD ICDs. 


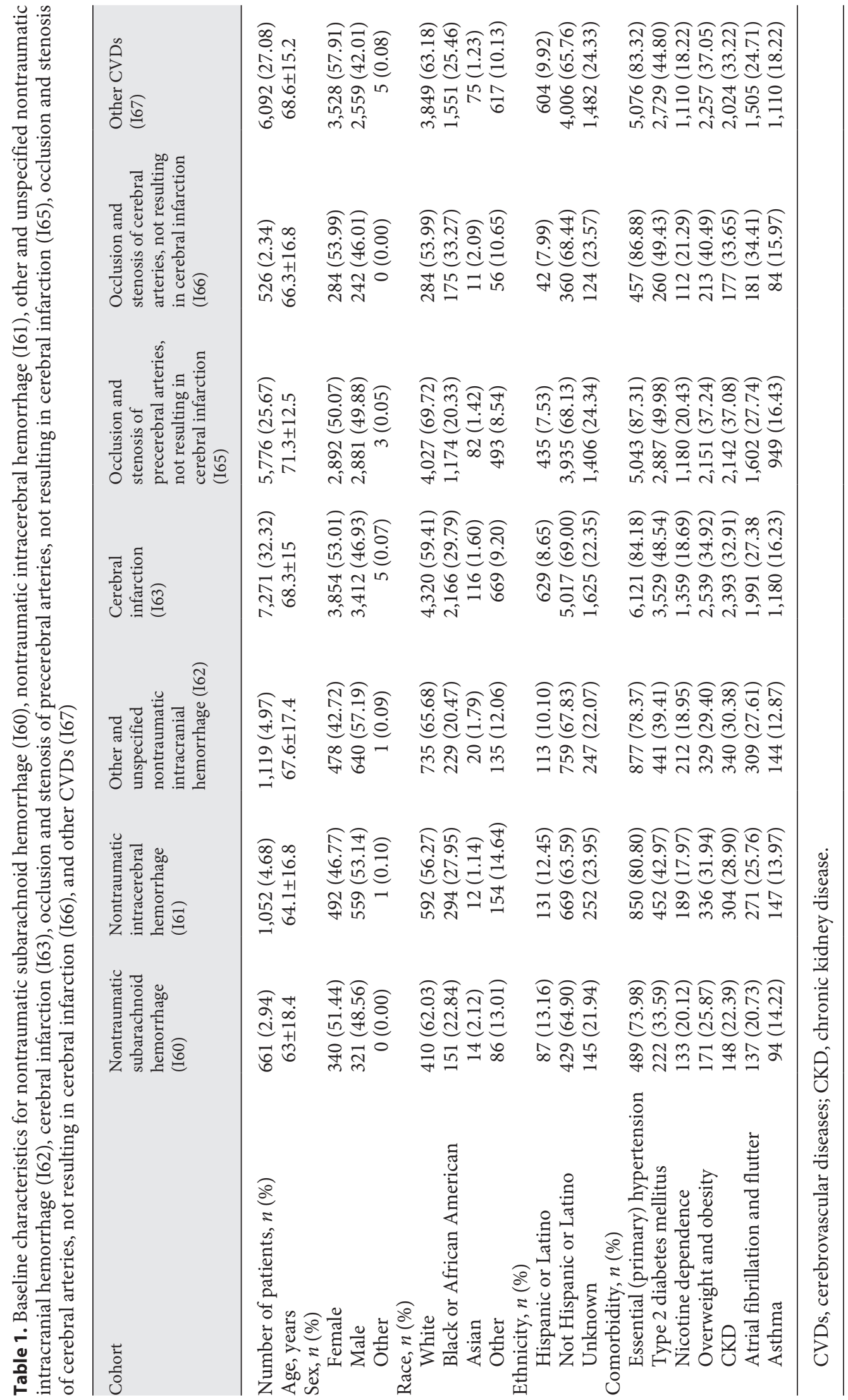



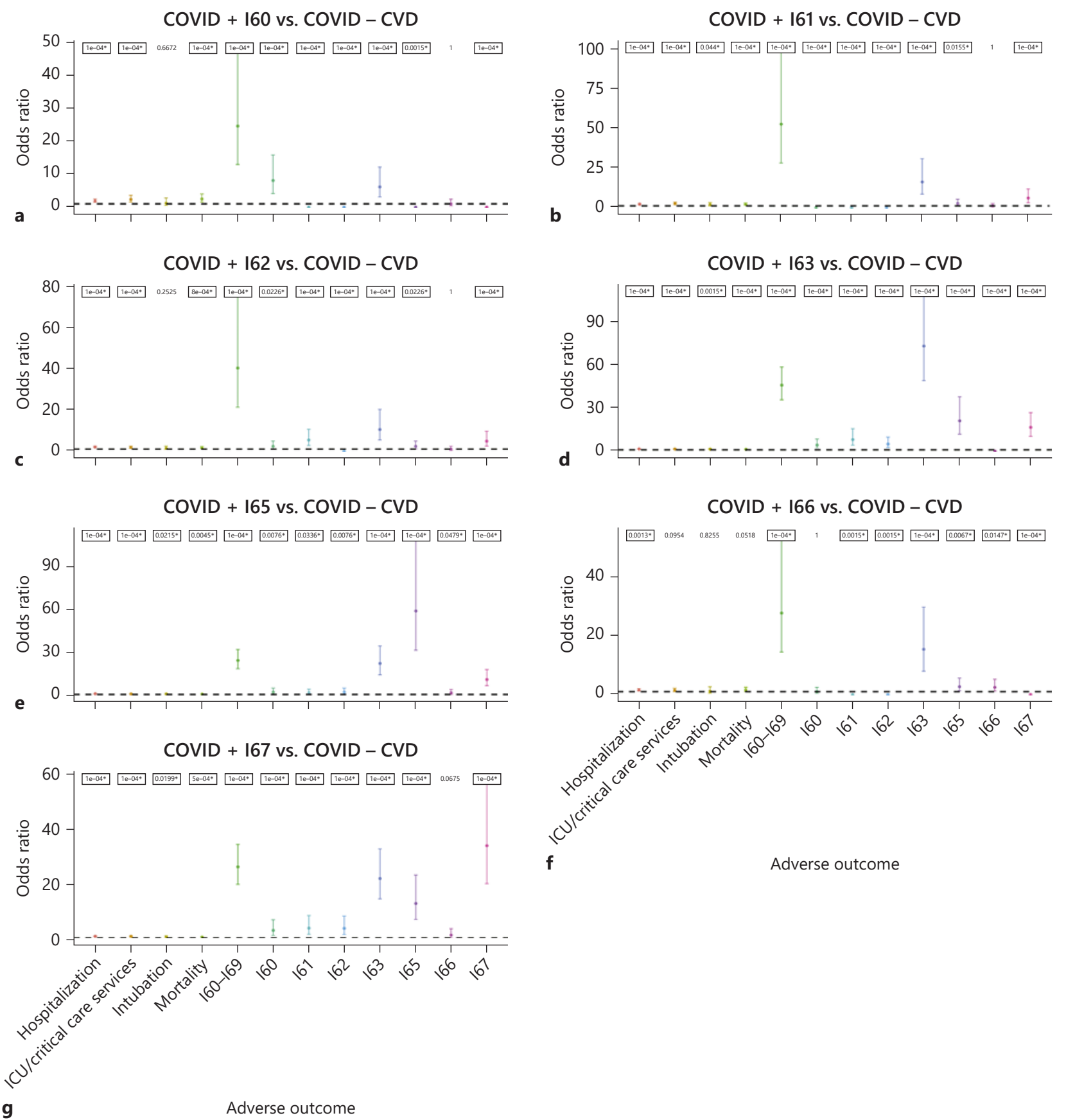

Adverse outcome

Fig. 1. Outcome analysis of COVID-19 + nontraumatic subarachnoid hemorrhage (I60) versus COVID-19 without any prior CVD (a), COVID-19 + nontraumatic intracerebral hemorrhage (I61) versus COVID-19 without CVD (b), COVID-19 + other and unspecified nontraumatic intracranial hemorrhage (I62) versus COVID-19 without CVD (c), COVID-19 + cerebral infarction (I63) versus COVID-19 without CVD (d), COVID-19+ occlusion and stenosis of precerebral arteries, not resulting in cerebral infarction (I65) versus COVID-19 without CVD (e), COVID-19 + occlusion and stenosis of cerebral arteries, not resulting in cerebral infarction
(I66) versus COVID-19 without CVD (f), and COVID-19 + other CVD (I67) versus COVID-19 without a CVD (g). The panels depict OR for adverse outcomes and subsequent CVD events of each comparison group. The error bars represent 95\% CI of the ORs. The numbers above the graph represent the $p$ values for each group as calculated by the z-test based on the risk difference. All significant $p$ values are denoted by * inside a box. CVDs, cerebrovascular diseases; ORs, odds ratios; CI, confidence interval; COVID-19, coronavirus disease 2019. 


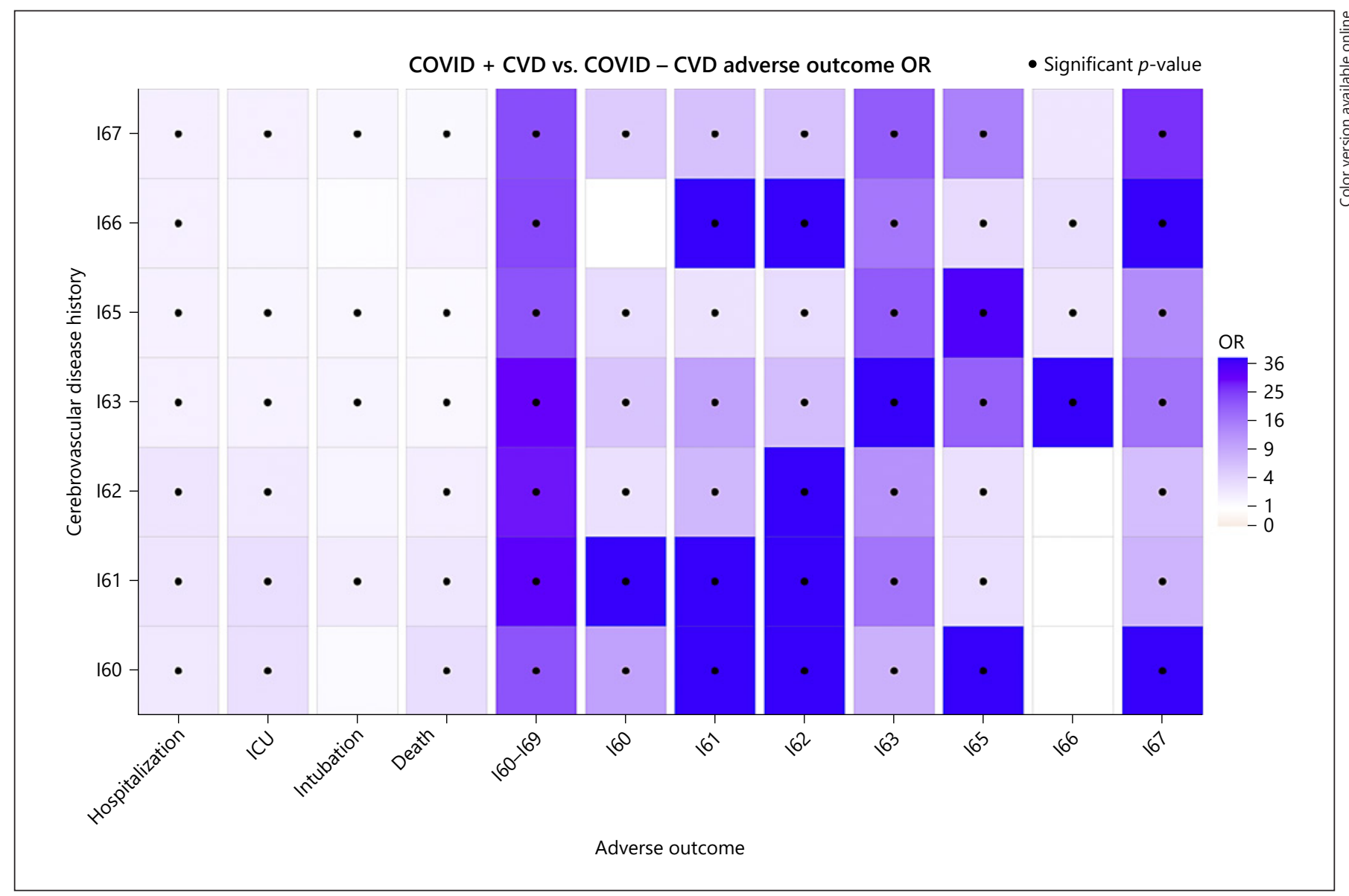

Fig. 2. Heatmap of OR for adverse outcomes for individual ICDs with COVID-19 versus COVID-19 without any history of CVD. The CVD ICDs include any CVD (I60-I69), nontraumatic subarachnoid hemorrhage (I60), nontraumatic intracerebral hemorrhage (I61), other and unspecified nontraumatic intracranial hemorrhage (I62), cerebral infarction (I63), occlusion and stenosis of precerebral arteries, not resulting in cerebral infarction (I65), oc-

\section{Outcome Analysis}

The results of all adverse outcome analyses for patients with individual ICDs and COVID-19, compared to a matched control COVID-19 cohort without any CVD are shown in Figure 1 as the OR with the error bars representing $95 \%$ confidence interval (CI), and the risk difference $p$ values are reported. For better visualization, Figure 2 shows a heatmap of the ORs as reported in Figure 1 with significant outcomes denoted. The associated details and numbers are described below and can be found in Table 2 and online suppl. Tables 1-7; for all online suppl. material, see www.karger.com/doi/10.1159/000517499. Patient counts of $1-10$ are reported as 10 by TriNetX to obfuscate patient and $\mathrm{HCO}$ identity. clusion and stenosis of cerebral arteries, not resulting in cerebral infarction (I66), and other CVDs (I67). The colors represent the magnitudes of the ORs. Significant $p$ values $(\leq 0.05)$ as calculated by the z-test based on the risk difference are represented by a bold circle. CVDs, cerebrovascular diseases; ORs, odds ratios; COVID-19, coronavirus disease 2019.

\section{Hospitalization, ICU/Critical Care Services,} Intubation, and Mortality Associated to COVID-19

A prior diagnosis of most CVDs within 10 years of a COVID-19 diagnosis significantly increases the patient's risk of hospitalization, ICU care, intubation, and mortality. There are no CVD cohorts, where the risk of adverse outcomes is lower in the propensity-matched control cohort within 90 days of a COVID-19 diagnosis reported statistically significant increases in risk difference to have a z-test $p$ value of $\leq 0.05$ (online suppl. Tables $1-7$ ). The ORs for hospitalization range between 1.5 (95\% CI: 1.41.6) for I65 and 2.1 (95\% CI: 1.7-2.5) for I61, with all CVD cohorts having a statistically significant risk increase. The ORs for ICU/critical care services range between 1.4 (95\% 


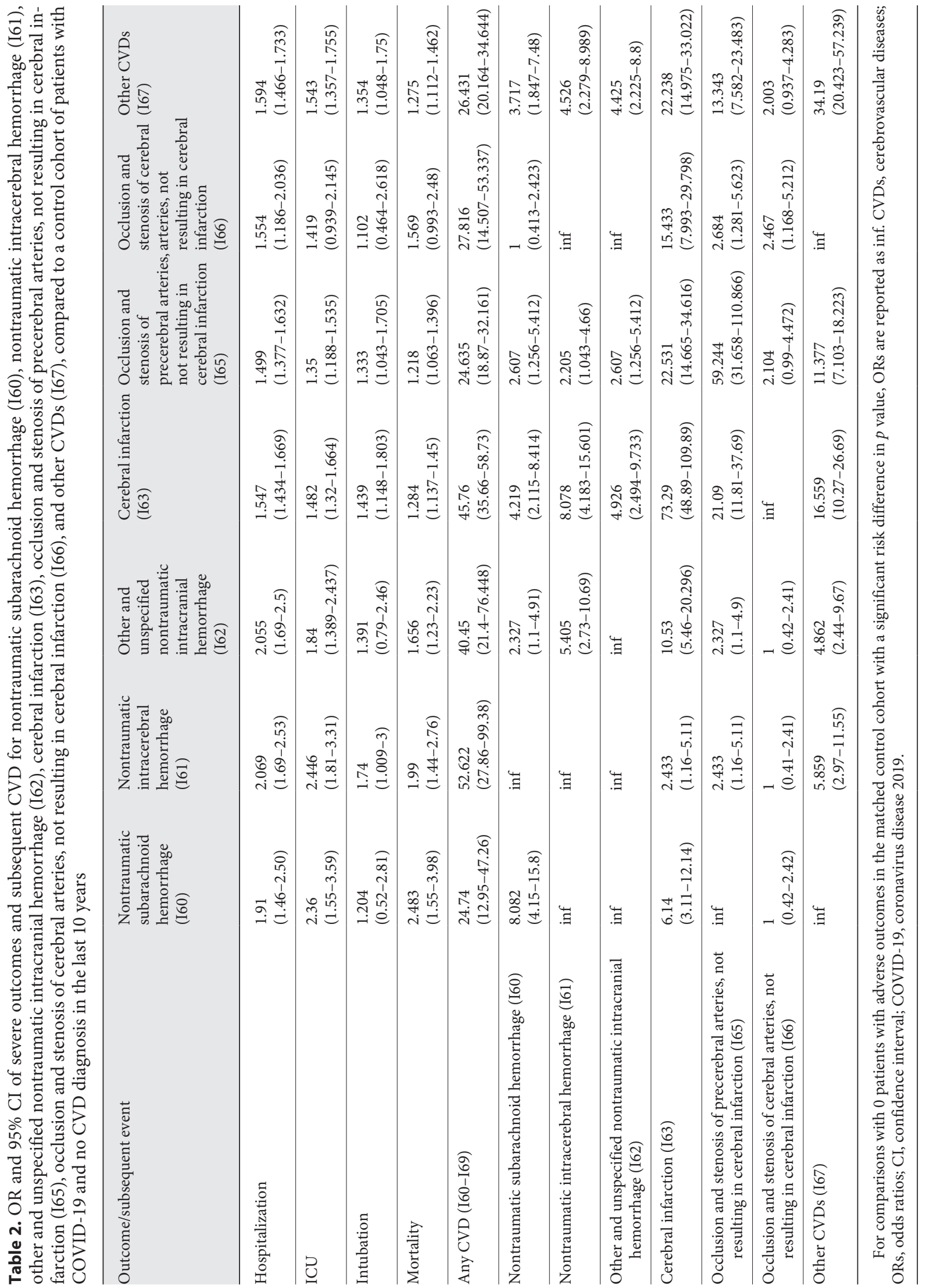


CI: 1.2-1.5) for I65 and 2.5 (95\% CI: 1.8-3.3) for I61. Except for I66 (OR 1.4, 95\% CI: 0.9-2.1), all other CVD cohorts have statistically significant risk increases. The ORs for intubation range between 1.1 (95\% CI: 0.5-2.6) for I66 and 1.7 (95\% CI: 1.01-3) for I61. Patients with a previous diagnosis of I61, I63, I65, and I67 had a significantly increased risk for intubation within 90 days of COVID-19 diagnosis. The ORs for mortality range between $1.2(95 \%$ CI: 1.06-1.4) for I65 and 2.5 (95\% CI: 1.6-4.0) for I60. Except for I66 (OR 1.6, 95\% CI: 0.99-2.5), all other CVD cohorts have a statistically significant risk increase in mortality. The highest ORs for mortality were for I60 and I61.

\section{Subsequent CVD Events Associated with COVID-19}

Patients with a prior diagnosis of CVD are at markedly increased risk of subsequent CVD events within 90 days of a COVID-19 diagnosis. There are no CVD cohorts, where the risk of the subsequent CVD events is lower in the propensity-matched control cohort (online suppl. Tables 1-7). Patients in the CVD cohorts are at the highest risk for subsequent cerebral infarctions (I63), with all CVD cohorts at a significantly increased risk. The ORs for a subsequent cerebral infarction range between 2.4 (95\% CI: 1.2-5.1) for patients with prior I61 and 73.3 (95\% CI: 48.9-109.9) for patients with a history of cerebral infarction. All CVD cohorts are also at a significantly increased risk for nontraumatic intracerebral and unspecified intracranial hemorrhage (I61, I62) and other CVDs (I67).

\section{Discussion}

We evaluated the baseline characteristics and adverse outcomes of patients who had COVID-19 and a diagnosis of a CVD within the past 10 years prior to COVID-19 diagnosis. All patient cohorts with previous CVD diagnosis, with the exception of occlusion and stenosis of cerebral arteries (I66), had increased the risk of hospitalization, ICU/critical care services, and mortality. Kummer et al. [9] reported increased inhospital mortality of OR = 1.28 in COVID patients with a prior diagnosis of stroke compared to control. This is in-line with our 90 -day mortality for cerebral infarction (i.e., ischemic stroke) of OR $=1.28$. In particular, the mortality for all cohorts except I66 was significantly increased with ORs 1.3-2.5 compared to the matched control COVID group with no prior CVD. Our study demonstrates that propensitymatched control for baseline characteristics and common comorbidities and the risks of the noted adverse outcomes within 90 days of COVID-19 infection are yet significantly increased in patients with any history of CVD prior to COVID-19.

Although previous studies have indicated that $\mathrm{CO}$ VID-19 could cause neurological disorders [8, 19, 20], it is unclear why the adverse outcomes in these patients with previous CVD diagnoses are more common. Perhaps a prior CVD can trigger long-lasting cerebrovascular damage and immune suppression that would result in increased susceptibility for adverse outcomes. Although the mechanism in which these long-lasting changes and immune suppression occurs is not well understood, a recent study has shown extrinsic T-cell apoptosis through inflammasome activation in myeloid cells and FasL expression on monocytes as the main driver of lymphopenia and secondary immunosuppression after stroke [21]. Patients in the I61 cohort with nontraumatic intracerebral hemorrhage are at increased risk for most subsequent CVD. The microbleeds and hemorrhagic lesions that result in intracerebral bleeding can lead to generalized endothelialitis, which could increase susceptibility for disseminated intravascular coagulation and a prothrombotic state [22, 23]. Additionally, patients with SARS-CoV-2 have been found to have low platelet levels, which in the setting of a previous intracerebral hemorrhage and subsequent endothelial changes can significantly contribute to the prothrombotic and inflammatory states of COVID-19 [24-29]. The risk of new CVD recurrence within 90 days of COVID-19 diagnosis was much higher than a newly diagnosed CVD in COVID-19 patients without any prior CVD episode. These findings support the concept that cerebrovascular dysfunction can lead to long-lasting changes that make these individuals remarkably vulnerable to the neuroinvasive properties of the SARS-CoV-2 virus.

The markedly elevated odds of adverse outcomes in patients with previous CVD are an important finding, given that, they comprise $6.09 \%$ of the reported COVID-19 cases within the TriNetX research network. Additionally, these findings raise the possibility that not all cerebrovascular manifestations observed in patients with COVID-19, especially in critically ill patients, are directly caused by COVID-19 but rather mediated by chronic inflammatory hyperactivation from previous CVD that makes these patients prone to adverse events. The findings in this study, derived from a large sample size, utilized propensity score matching, and the real-world nature of the data provides valuable information for health- 
care providers to be especially diligent about identifying these patients at the time of admission and use specific precautions and screening protocols for timely recognition and treatment of subsequent CVD.

The study has several limitations due to the structure of the available data and other factors. The analysis does not take into consideration the number of instances a patient experienced CVD in the past. Additionally, it is not possible to assess if a specific patient may have had a secondary COVID-19 infection during the 90-day period from the first infection; however, we expect those rates to be similar between the 2 cohorts. Despite using appropriate propensity matching, there is still a possibility of some unknown confounding factors, such as those related to access to medical care, time at which patients sought medical care, and other socioeconomic factors that are not available in the electronic health network system. As a result, these findings may not be generalizable to other patient populations. Additionally, different HCOs may have different outcomes based on the availability of resources and different management strategies, which could not be accounted for during patient selection due to data privacy restrictions.

This study attempts to isolate a prior history of CVD as the main independent factor in the observed increase of negative outcomes for patients with a prior history of CVD. However, future analyses may benefit from utilizing a larger range of comorbidities, types of prescribed medications, other recent hospitalizations, and most recent laboratory results in the propensity matching as these factors may elucidate additional factors of contribution to COVID-19 outcomes.

\section{Conclusion}

CVDs have been shown to occur commonly in patients with COVID-19 diagnosis, which could have multifactorial etiology. Patients diagnosed with any prior CVD in the past 10 years and COVID-19 are at significantly higher morbidity and mortality as well as subsequent CVD within 90 days of COVID-19 diagnosis. Patients with a prior nontraumatic subarachnoid/intracerebral hemorrhage and cerebral infarction are at the highest risk for various subsequent CVD. These findings have immediate implications in identifying patients with the aforementioned diagnoses. It is important to note that the risk factor identification/stratification in the setting of hospital admission for COVID-19 symptoms could often be delayed or missed, particularly in peak phases of the outbreak. These challenges could be responsible for the underestimation of COVID-19-related CVD, and inadequate stroke monitoring could lead to irreversible damages and result in increased length of hospital stay and poor prognosis.

\section{Statement of Ethics}

The data presented in this study as provided by TriNetX. TriNetX is compliant with the Health Insurance Portability and Accountability Act (HIPAA), the US federal law which protects the privacy and security of health-care data. TriNetX is certified to the ISO 27001:2013 standard and maintains an information security management system to ensure the protection of the health-care data, and it has access to and to meet the requirements of the HIPAA security rule. Any data displayed on the TriNetX platform in aggregate form, or any patient level data provided in a data set generated by the TriNetX platform, only contains de-identified data as per the de-identification standard defined in section $\$ 164.514$ (a) of the HIPAA privacy rule. The process by which the data are de-identified is attested to through a formal determination by a qualified expert, as defined in section $\$ 164.514$ (b) (1) of the HIPAA privacy rule. This study did not require additional local ethical approval in accordance with local/national guidelines. Written informed consent from participants was not required for the study presented in this article in accordance with local/national guidelines.

\section{Conflict of Interest Statement}

The authors have no conflicts of interest to declare.

\section{Funding Sources}

The data access was supported by NIH grant TR001439 and Clinical and Translational Science Award (UL1).

\section{Author Contributions}

A.M.N.: designed the study, acquired, analyzed, and drafted the manuscript. All authors interpreted the data, critically revised, and reviewed the manuscript. P.K.: supervised the study and approved the final version of the manuscript. All authors consent to publication.

\section{Availability of Data and Material}

Data for this study are not publicly available because of a data use agreement. For requests to access the study data, contact the corresponding author. 


\section{References}

1 World Health Organization. Coronavirus disease (COVID-19) pandemic.

2 Paniz Mondolfi A, Bryce C, Grimes Z, Gordon RE, Reidy J, Lednicky J, et al. Central nervous system involvement by severe acute respiratory syndrome coronavirus 2 (SARS CoV 2). J Med Virol. 2020;92(7):699-702.

3 Baig AM, Khaleeq A, Ali U, Syeda H. Evidence of the COVID-19 virus targeting the CNS: tissue distribution, host-virus interaction, and proposed neurotropic mechanisms. ACS Chem Neurosci. 2020;11(7):995-8.

4 Daneshi SA, Taheri M, Fattahi A. SARS coronavirus 2 and central nervous system manifestations: causation, relation, or coexistence? A case series study and literature review. $\mathrm{Br} J$ Neurosurg. 2020 Dec 21:1-6.

5 Lee MH, Perl DP, Nair G, Li W, Maric D, Murray $\mathrm{H}$, et al. Microvascular injury in the brains of patients with Covid-19. N Engl J Med. 2020 Dec 30;384(5):481-3.

6 Siow I, Lee KS, Zhang JJY, Saffari SE, Ng A, Young B. Stroke as a neurological complication of COVID-19: a systematic review and meta-analysis of incidence, outcomes and predictors. J Stroke Cerebrovasc Dis. 2021 Dec 15;30(3):105549.

7 Yamaoka-Tojo M. Vascular endothelial glycocalyx damage in COVID-19. Int J Mol Sci. 2020 Dec 19;21(24):21.

8 Mao L, Jin H, Wang M, Hu Y, Chen S, He Q, et al. Neurologic manifestations of hospitalized patients with coronavirus disease 2019 in Wuhan, China. JAMA Neurol. 2020;77(6): 683-90.

9 Kummer BR, Klang E, Stein LK, Dhamoon MS, Jetté N. History of stroke is independently associated with in-hospital death in patients with COVID-19. Stroke. 2020;51(10) 3112-4.

10 Offner H, Subramanian S, Parker SM, Afentoulis ME, Vandenbark AA, Hurn PD. Experimental stroke induces massive, rapid activation of the peripheral immune system. J Cereb Blood Flow Metab. 2006;26(5):654-65.

11 Schimmel SJ, Acosta S, Lozano D. Neuroinflammation in traumatic brain injury: a chronic response to an acute injury. Brain Circ. 2017;3(3):135.
12 National Center for Health Statistics (NCHS), Centers for Medicare and Medicaid Services (CMS). ICD-10-CM official guidelines for coding and reporting; 2021.

13 Chen N, Zhou M, Dong X, Qu J, Gong F, Han $\mathrm{Y}$, et al. Epidemiological and clinical characteristics of 99 cases of 2019 novel coronavirus pneumonia in Wuhan, China: a descriptive study. Lancet. 2020;395(10223):507-13.

14 de Lusignan S, Dorward J, Correa A, Jones N, Akinyemi $\mathrm{O}$, Amirthalingam $\mathrm{G}$, et al. Risk factors for SARS-CoV-2 among patients in the Oxford Royal College of general practitioners research and surveillance centre primary care network: a cross-sectional study. Lancet Infect Dis. 2020 Sep;20(9):1034.

15 Williamson EJ, Walker AJ, Bhaskaran K, Bacon S, Bates C, Morton CE, et al. Factors associated with COVID-19-related death using opensafely. Nature. 2020;584(7821):430-6.

16 Zhang J, Dong X, Cao Y, Yuan YD, Yang YB, Yan Y, et al. Clinical characteristics of $140 \mathrm{pa}$ tients infected with SARS CoV 2 in Wuhan, China. Allergy. 2020;75(7):1730.

17 Austin PC. An introduction to propensity score methods for reducing the effects of confounding in observational studies. Multivariate Behav Res. 2011 May;46(3):399-424.

18 Von Elm E, Altman DG, Egger M, Pocock SJ, Gøtzsche PC, Vandenbroucke JP. The strengthening the reporting of observational studies in epidemiology (STROBE) statement: guidelines for reporting observational studies. Ann Intern Med. 2007;147(8):573-7.

19 Jin H, Hong C, Chen S, Zhou Y, Wang Y, Mao $\mathrm{L}$, et al. Consensus for prevention and management of coronavirus disease 2019 (COVID-19) for neurologists. Stroke Vasc Neurol. 2020 Jun;5(2):146-51.

20 Liang WH, Guan WJ, Li CC, Li YM, Liang $\mathrm{HR}$, Zhao Y, et al. Clinical characteristics and outcomes of hospitalised patients with $\mathrm{CO}$ VID-19 treated in Hubei (epicentre) and outside Hubei (non-epicentre): a nationwide analysis of China. Eur Respir J. 2020;55(6): 2000562.
21 Roth S, Cao J, Singh V, Tiedt S, Hundeshagen G, Li T, et al. Post-injury immunosuppression and secondary infections are caused by an AIM2 inflammasome-driven signaling cascade. Immunity. 2021;54(4):648-e8.

22 Ackermann M, Verleden SE, Kuehnel M, Haverich A, Welte T, Laenger F, et al. Pulmonary vascular endothelialitis, thrombosis, and angiogenesis in Covid-19. N Engl J Med. 2020 Jul 9;383(2):120-8.

23 Coolen T, Lolli V, Sadeghi N, Rovai A, Trotta $\mathrm{N}$, Taccone FS, et al. Early postmortem brain MRI findings in COVID-19 non-survivors. MedRxiv. 2020.

24 Al Saiegh F, Ghosh R, Leibold A, Avery MB, Schmidt RF, Theofanis T, et al. Status of SARS-CoV-2 in cerebrospinal fluid of patients with COVID-19 and stroke. J Neurol Neurosurg Psychiatry. 2020 Aug;91(8):8468.

25 Beyrouti R, Adams ME, Benjamin L, Cohen H, Farmer SF, Goh YY, et al. Characteristics of ischaemic stroke associated with COVID-19. J Neurol Neurosurg Psychiatry. 2020 Aug;91(8):889-91.

26 Fraiman P, Godeiro Junior C, Moro E, Cavallieri F, Zedde M. COVID-19 and Cerebrovascular diseases: a systematic review and perspectives for stroke management. Front Neurol. 2020;11(1153):574694.

27 González-Pinto T, Luna-Rodríguez A, Moreno-Estébanez A, Agirre-Beitia G, RodríguezAntigüedad A, Ruiz-Lopez M. Emergency room neurology in times of COVID-19: malignant ischaemic stroke and SARS-CoV-2 infection. Eur J Neurol. 2020 Sep;27(9):e356.

28 Li Y, Li M, Wang M, Zhou Y, Chang J, Xian $\mathrm{Y}$, et al. Acute cerebrovascular disease following COVID-19: a single center, retrospective, observational study. Stroke Vasc Neurol. 2020 Sep;5(3):279-84

29 Oxley TJ, Mocco J, Majidi S, Kellner CP, Shoirah H, Singh IP, et al. Large-Vessel stroke as a presenting feature of Covid-19 in the young. N Engl J Med. 2020 May 14;382(20):e60. 